\title{
A Diminished Role for Hydrogen Bonds in Antifreeze Protein Binding to Ice ${ }^{\dagger}$
}

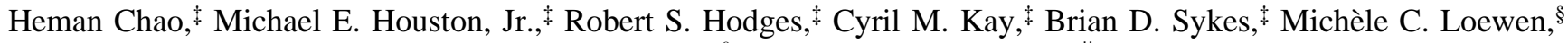 \\ Peter L. Davies, ${ }^{*} \S$ and Frank D. Sönnichsen" \\ Protein Engineering Network of Centres of Excellence and Department of Biochemistry, University of Alberta, Edmonton, \\ Alberta T6G 2S2, Canada, Department of Biochemistry, Queen's University, Kingston, Ontario K7L 3N6, Canada, and \\ Department of Physiology and Biophysics, Case Western Reserve University, Cleveland, Ohio 44106-4970
}

Received April 8, 1997; Revised Manuscript Received August 11, $1997^{\circledR}$

\begin{abstract}
The most abundant isoform (HPLC-6) of type I antifreeze protein (AFP') in winter flounder is a 37-amino-acid-long, alanine-rich, $\alpha$-helical peptide, containing four Thr spaced 11 amino acids apart. It is generally assumed that HPLC-6 binds ice through a hydrogen-bonding match between the Thr and neighboring Asx residues to oxygens atoms on the $\{20 \overline{2} 1\}$ plane of the ice lattice. The result is a lowering of the nonequilibrium freezing point below the melting point (thermal hysteresis). HPLC-6, and two variants in which the central two Thr were replaced with either Ser or Val, were synthesized. The Ser variant was virtually inactive, while only a minor loss of activity was observed in the Val variant. CD, ultracentrifugation, and NMR studies indicated no significant structural changes or aggregation of the variants compared to HPLC-6. These results call into question the role of hydrogen bonds and suggest a much more significant role for entropic effects and van der Waals interactions in binding AFP to ice.
\end{abstract}

Type $\mathrm{AFP}^{1}$ is the smallest and arguably the simplest of the four macromolecular antifreeze types characterized to date (1). It is in effect a single, long $\alpha$-helix and therefore lacks tertiary structure (2). The most abundant isoform of this AFP (HPLC-6) from winter flounder (Pleuronectes americanus) is 37 amino acids long, contains three complete 11-amino-acid repeats of Thr- $\mathrm{X}_{2}-\mathrm{AsX}-\mathrm{X}_{7}$, where $\mathrm{X}$ is generally alanine, and ends with the start of a fourth repeat. This helical periodicity that places the Thr and Asx residues on the same face of the helix, suggested a mechanism for adsorption of the AFP to ice in which these regularly spaced hydrophilic groups would hydrogen bond to oxygen atoms in the ice lattice (3). Adsorption leads to inhibition of ice crystal growth (4) because ice is forced to grow with a surface curvature between the bound AFP, which in turn results in a lowering of the nonequilibrium freezing point below the melting point $(5,6)$. The difference in these two temperatures is termed thermal hysteresis and is used as a measure of antifreeze activity.

At very low concentrations, AFP bind to ice but do not stop its growth. Under these conditions bound AFP is frozen into the ice rather than excluded by the advancing ice front. The protein binding planes in these crystals have been made visable by sublimation (ice etching) and determined to be

\footnotetext{
$\dagger$ This work is supported by the Protein Engineering Network of Centres of Excellence of Canada and A/F Protein, Inc. F.D.S. acknowledges support from NIH Grant GM55362.

* Author to whom correspondence should be addressed: Peter L. Davies, Department of Biochemistry, Queen's University, Kingston, Ontario K7L 3N6, Canada. Tel: (613) 545-2983. Fax: (613) 545-2497. E-mail: daviesp@post.queensu.ca.

University of Alberta.

\& Queen's University.

"Case Western Reserve University.

${ }^{\otimes}$ Abstract published in Advance ACS Abstracts, November 15, 1997.

${ }^{1}$ Abbreviations: AFP, antifreeze protein; HPLC, high-performance liquid chromatography; $\mathrm{CD}$, circular dichroism; NMR, nuclear magnetic resonance; DQF-COSY, double-quantum-filtered correlation spectroscopy; PE-COSY, primitive exclusive correlation spectroscopy; TOCSY, total correlated spectroscopy; NOE, nuclear Overhauser effect; NOESY, nuclear Overhauser enhanced spectropscpy.
}

the $\{20 \overline{2} 1\}$ pyramidal plane of hexagonal ice $\left(I_{\mathrm{h}}\right)$ for type I AFP (5). Moreover, because this antifreeze is a nonglobular, extended molecule it was possible to establish a direction $\langle 01 \overline{1} 2\rangle$ of binding on the plane. An elegant proof of this resulted from the synthesis of an all D-type I AFP, which was shown by the ice etching method to bind to the same plane but in the mirror image direction (7). This information was used to suggest a hydrogen-bonding match between the $i, i+11$ threonines spaced $16.5 \AA$ apart along the helix and accessible ice lattice oxygens spaced $16.7 \AA$ apart along the $\langle 01 \overline{1} 2\rangle$ direction of the $\{20 \overline{2} 1\}$ binding plane.

On the basis of this match and the structure of the $\alpha$-helix, a number of models have been proposed to account for the selective and specific binding of type I AFP to ice that is the foundation of the adsorption-inhibition mechanism. Although all of these models share the Thr hydrogen-bonding match, there is no consensus about the role of Asx. In two reports these residues were not featured in the ice-binding model $(8,9)$; in others they were hydrogen bonded to different ranks of oxygen atoms in the lattice $(10,11)$ or they occupied a cage position within the lattice without hydrogen bonding (12).

Throughout all of these deliberations there has been concern that the number and strength of the available hydrogen bonds (with or without a contribution from the Asx residues) may be inadequate to explain tight binding of the antifreeze to ice. At least two imaginative ideas have been put forward to reinforce the hydrogen-bonding hypothesis by suggesting ways in which more hydrogen bonds can be brought to bear on the binding interaction. One suggestion, that the hydroxyl groups of the ice-binding Thr occupy lattice oxygen positions to form three hydrogen bonds instead of one (13), is, however, not compatible with the $\alpha$-helical structure of the AFP (11). The other suggestion is that type I AFP forms patches on the ice surface through interpeptide interactions resulting from side-by-side alignment of the helices. Thus a cluster of $n \alpha$-helices would be the operative unit, which would have a multiple of $n$ hydrogen bonds. 
Although still a formal possibility, this mode of interaction with ice is not supported by the simplest interpretation of the results of AFP mixing experiments, which is that each AFP acts independently (14).

Refinement of the X-ray crystal structure (11) has confused the situation in some respects. The designated ice-binding Thr are all reported to adopt a rigid side-chain conformation with a $\chi 1$ of $-60^{\circ}$. This is the least accessible rotamer for external hydrogen-bonding and one in which the hydroxyl groups are better positioned to form intra- than extrahelix bonds. It was postulated by these authors that the rigidity and common orientation of the Thr side chains are critical features for concerted hydrogen bonding of the AFP to ice. In contrast to this, solution NMR studies near the solution freezing point indicate that the polar side chains of type I AFP are in fact quite flexible (15). Although the central Thr (T13 and T24) have a preference for the $-60^{\circ}$ rotamer seen in the X-ray structure, all four Thr can and do sample many rotameric states, at least prior to binding to ice.

To explore the role of this amino acid in ice binding, we have replaced the two central Thr using chemical synthesis with either Ser to retain the hydoxyl group or Val to retain the methyl group. Surprisingly, the results suggest that hydrogen bonds make a relatively minor contribution to ice binding.

\section{MATERIALS AND METHODS}

Peptide Synthesis and Purification. Type I AFP and its variants were synthesized by solid-phase peptide synthesis methodology and purified as described previously $(15,16)$. The identity of the peptides and concentration of their solutions were assessed by amino acid analysis. Samples were hydrolyzed in $6 \mathrm{~N} \mathrm{HCl}$ at $160{ }^{\circ} \mathrm{C}$ for $1.5 \mathrm{~h}$ in sealed evacuated tubes. Hydrolysate was analyzed in a Beckman Model 6300 amino acid analyzer (San Ramon, CA). The amount of each identified amino acid was corrected by reference to an added norleucine standard.

Circular Dichroism Spectroscopy. CD spectra were recorded on a Jasco J-500C spectropolarimeter (Jasco, Easton, MD) as previously described (17). For temperature denaturation studies, the changes in the helical content of the sample was monitored at $222 \mathrm{~nm}$. Each data point was the average of at least 24 samplings. The buffer used was $50 \mathrm{mM}$ potassium phosphate ( $\mathrm{pH} 7.0), 50 \mathrm{mM} \mathrm{KCl}$.

Sedimentation Equilibrium Ultracentrifugation. Sedimentation experiments were performed on a Beckman model E analytical ultracentrifuge equipped with electronic speed control and Rayleigh interference optics. Samples were first dialyzed exhaustively against $50 \mathrm{mM}$ potassium phosphate (pH 7.0), $50 \mathrm{mM} \mathrm{KCl}$ at $4{ }^{\circ} \mathrm{C}$. A $100 \mu \mathrm{L}$ aliquot was loaded into the $12 \mathrm{~mm}$ double-sector, charcoal-filled Epon cell, and runs $(48 \mathrm{~h})$ with rotor speeds of $36 \mathrm{~K}$ and $40 \mathrm{~K} \mathrm{rpm}$ at $5{ }^{\circ} \mathrm{C}$ were performed. The initial loading concentration was 3.52 $\mathrm{mg} / \mathrm{mL}$ for the LSAAN samples and $3.48 \mathrm{mg} / \mathrm{mL}$ for the LVAAN runs. Equilibrium photographs were taken at the end of the run, and fringe counts were performed on a Nikon Model 6C microcomparator. The data were analyzed globally by the program NONLIN (nonlinear least squares analysis of sedimentation equilibrium data) of Johnson et al. (18). Using the program SEQSEE (19), the partial specific volumes of LSAAN and LVAAN were calculated to be 0.71 and $0.73 \mathrm{mg} / \mathrm{mL}$, respectively (20). The density of the solution was calculated to be $1.004 \mathrm{~g} / \mathrm{mL}$.
Antifreeze Activity and Photomicroscopy. Thermal hysteresis, defined as the temperature difference $\left({ }^{\circ} \mathrm{C}\right)$ between the melting point and the nonequilibrium freezing point of a solution, was measured using a nanoliter osmometer (Clifton Technical Physics, Hartford, NY) as described by Chakrabartty and Hew (21). For the purpose of comparison, an ice crystal growth rate of more than $0.2 \mu \mathrm{m} / \mathrm{s}$ is defined as having reached the solution freezing point (22). This operational definition was introduced to standardize thermal hysteresis measurements when it became apparent that some AFP mutants exhibit slow ice crystal growth prior to a defined freezing end-point, and others grow so rapidly that a distinguishable end-point is not observed. All measurements were made in $0.1 \mathrm{M} \mathrm{NH}_{4} \mathrm{HCO}_{3}(\mathrm{pH} 7.9)$ and $0.02 \%$ w/v sodium azide. Ice crystal morphology was observed through a Leitz dialux 22 microscope and recorded by a Panasonic CCTV camera linked to a JVC Super VHS video recorder. Still images were obtained from a Silicon Graphics INDY terminal using IRIS Capture version 1.2. For ice growth rate analysis, samples were held at a fixed degree of undercooling and images were captured at 0 and 10 min time points.

NMR Spectroscopy. For the NMR-spectroscopic experiments, $6 \mathrm{mg}$ of LSAAN and LVAAN peptide each were dissolved in $90 \% / 10 \% \mathrm{H}_{2} \mathrm{O} / \mathrm{D}_{2} \mathrm{O}(500 \mu \mathrm{L})$ or in $\mathrm{D}_{2} \mathrm{O}$, and the $\mathrm{pH}$ was adjusted to 7.3/7.2. All experiments were acquired on a Varian Unityplus spectrometer operating at a ${ }^{1} \mathrm{H}$ frequency of $600 \mathrm{MHz}$ and were processed using VNMR 5.1 on a Silicon Graphics workstation. Sample temperature settings were calibrated with a methanol sample, and all experiments were performed at an actual temperature of 4 ${ }^{\circ} \mathrm{C}$. 2D-Homonuclear NMR experiments in $\mathrm{H}_{2} \mathrm{O}$ included a DQF-COSY $(23,24)$, NOESY spectra $(25,26)$ with mixing times from 100 to $300 \mathrm{~ms}$ and a TOCSY experiment (27) using a dipsi-2 spinlock field with $68 \mathrm{~ms}$ mixing time. The DQF-COSY experiment used a presaturation pulse of $1.5 \mathrm{~s}$ for water supression, while the latter two experiments included watergate solvent supression (28). Experiments were generally performed using a sweep width of $6600 \mathrm{~Hz}$ and $1 \mathrm{~K}$ complex data points, and $5600 \mathrm{~Hz}, 400$ complex increments and quadrature detection via the States method in the direct and indirect dimension, respectively. The data were zero filled to yield spectra of $2 \mathrm{~K} \times 2 \mathrm{~K}$ complex data after transformation.

A PE-COSY experiment (29) was performed in $\mathrm{D}_{2} \mathrm{O}$ without solvent saturation with a repetition delay of $1.5 \mathrm{~s}$ and sweep widths of $3000 \mathrm{~Hz}$ and $2 \mathrm{~K} \times 350$ complex datapoints. The spectrum was zero-filled to $4 \mathrm{~K} \times 2 \mathrm{~K}$ datapoints and transformed using $45^{\circ}$ and $70^{\circ}$ shifted sinebell window functions. A DQF-COSY with a $1.5 \mathrm{~s}$ presaturation delay, 20-fold oversampling, digital filtering and $880 \mathrm{~Hz}$ frequency shifting (to place the center of the spectrum in the aliphatic region) during downsampling to $1 \mathrm{~K}$ complex data points in the direct dimension and a $1340 \mathrm{~Hz}$ sweep width and 400 complex data points in the indirect dimension was also acquired. The spectrum was transformed using zero-filling, shifted sinebell weighting functions, and frequency shifting by $1400 \mathrm{~Hz}$ in the indirect dimension to yield a final data set of $4 \mathrm{~K} \times 2 \mathrm{~K}$ data points. Coupling constants were determined and correlated with side chain rotamer populations as recently described (15). $\mathrm{C} \alpha \mathrm{H} / \mathrm{C} \beta \mathrm{H}$ coupling constants for Val and Thr residues were determined from the PE COSY and DQF-COSY experiments with the VNMR 


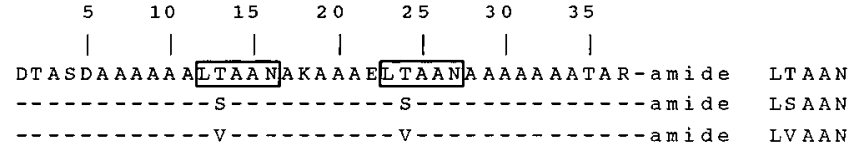

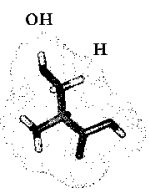

Ser

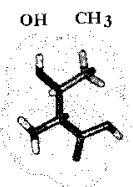

Thr

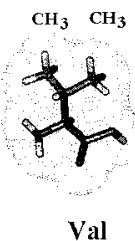

Val
FIGURE 1: Sequences of wild-type AFP and its variants (top) and schematic representation of the amino acid Ser, Thr and Val (bottom). (Top) The duplicate "ice-binding motifs" are boxed in the wild-type sequence (LTAAN). The variants are named according to their equivalent sequences. Amino acids that are unchanged in the variants as compared to the wild-type are indicated by a dash (-). All synthetic AFPs have a free N-terminus and an amidated C-terminus as found in the natural protein from fish serum. (Bottom) The atoms of the amino acids are displayed in "stick" form, the calculated solvent surface is represented by "point cloud" and atoms pertinent to the discussion are labeled as $\mathrm{H}$ for proton, $\mathrm{OH}$ for hydroxyl, and $\mathrm{CH}_{3}$ for methyl.

Table 1: Summary of CD Data for Type I AFP and Variants

\begin{tabular}{ccc}
\hline sample & $\begin{array}{c}{[\Theta]_{222 \mathrm{~nm}^{a}}} \\
\left(\mathrm{deg} \cdot \mathrm{cm}^{2} \cdot \mathrm{dmol}^{-1}\right)\end{array}$ & $T_{\mathrm{m}}{ }^{b}\left({ }^{\circ} \mathrm{C}\right)$ \\
\hline LTAAN & 39370 & 21 \\
LSAAN & 40533 & 24 \\
LVAAN & 40860 & 25 \\
\hline
\end{tabular}

${ }^{a}$ The observed molar ellipticity at $1{ }^{\circ} \mathrm{C} .{ }^{b}$ Apparent $T_{\mathrm{m}}$ was calculated assuming the observed ellipticity at $1{ }^{\circ} \mathrm{C}$ was essentially $100 \%$ (expected theoretical value is near $38000 \mathrm{deg} \cdot \mathrm{cm}^{2} \cdot \mathrm{dmol}^{-1}(39)$ ), and the observed ellipticity value at $60{ }^{\circ} \mathrm{C}$ was taken as the approximation for random coil.

software. Traces of the respective cross peaks in the direct dimension were extracted from the processed 2D data sets, and after an inverse Fourier transform retransformed with zero-filling to yield $64 \mathrm{~K}$ data point frequency domains. The signals were then fitted to 4 (due to partial overlap of V13 and V24 cross peaks) and 2 lines of Lorentzian shape, respectively. Additionally, the $\mathrm{Val} \mathrm{C} \alpha \mathrm{H} / \mathrm{C} \beta \mathrm{H}$ coupling constants were confirmed by curve fitting the respective resonances directly in a $1 \mathrm{D}-{ }^{1} \mathrm{H}-\mathrm{NMR}$ spectrum.

\section{RESULTS}

Helicity of the Type I AFP Variants LSAAN and LVAAN. Duplicate substitutions made in the two central ice-binding motifs (11), which changed the putative ice-binding residues T13 and T24 to Ser or Val (Figure 1), were predicted to have no serious impact on the helicity of the AFP. The CD $(190-250 \mathrm{~nm})$ profiles of these peptides were very similar at low temperatures with appropriate maximum and minima observed at regions near 190, 208, and $222 \mathrm{~nm}$ that are characteristic of a full helical structure (data not shown). Both variants were close to fully helical at $1{ }^{\circ} \mathrm{C}$ and had $T_{\mathrm{m}}$ values of $24{ }^{\circ} \mathrm{C}$ (LSAAN) and $25^{\circ} \mathrm{C}$ (LVAAN) that were slightly above that of the wild type $\left(21^{\circ} \mathrm{C}\right)$ (Table 1 , Figure 2$)$. These modest increases in $T_{\mathrm{m}}$ values are in line with the accepted helical propensities of amino acids which indicate that Ser and Val are less destabilizing than $\operatorname{Thr}(30,31)$.

Antifreeze Activity of the Type I AFP Variants LSAAN and $L V A A N$. The thermal hysteresis activity of synthetic wild-

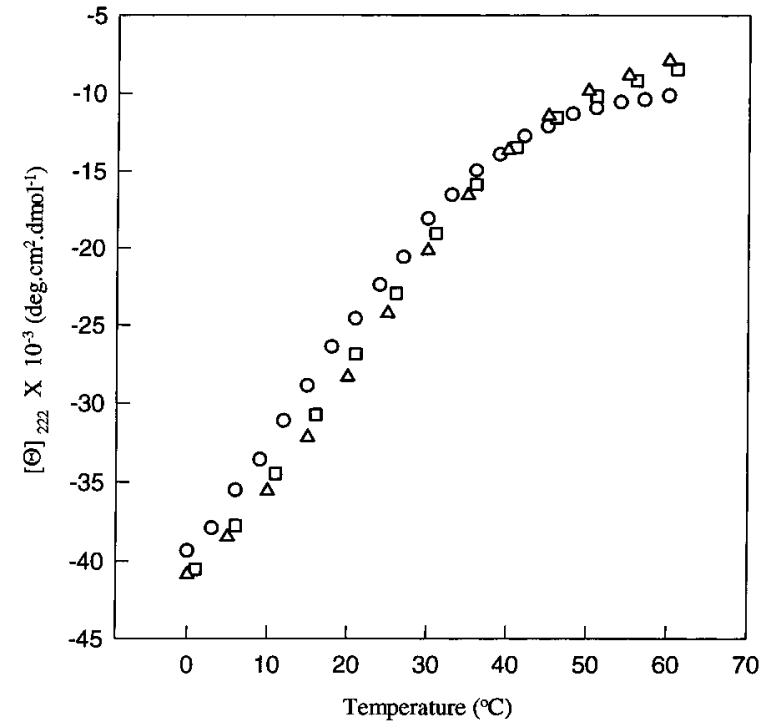

FIGURE 2: Reversible temperature-induced denaturation of type I AFP and its variants. The change in helical content of type I AFP $(\bigcirc)$, LSAAN $(\square)$, and LVAAN $(\triangle)$ was monitored at $222 \mathrm{~nm}$. The experiments were performed in a $50 \mathrm{mM}$ phosphate $(\mathrm{pH} 7.0), 50$ $\mathrm{mM} \mathrm{KCl}$ buffer. The protein concentration was $\sim 1 \mathrm{mg} / \mathrm{mL}$ for each sample.

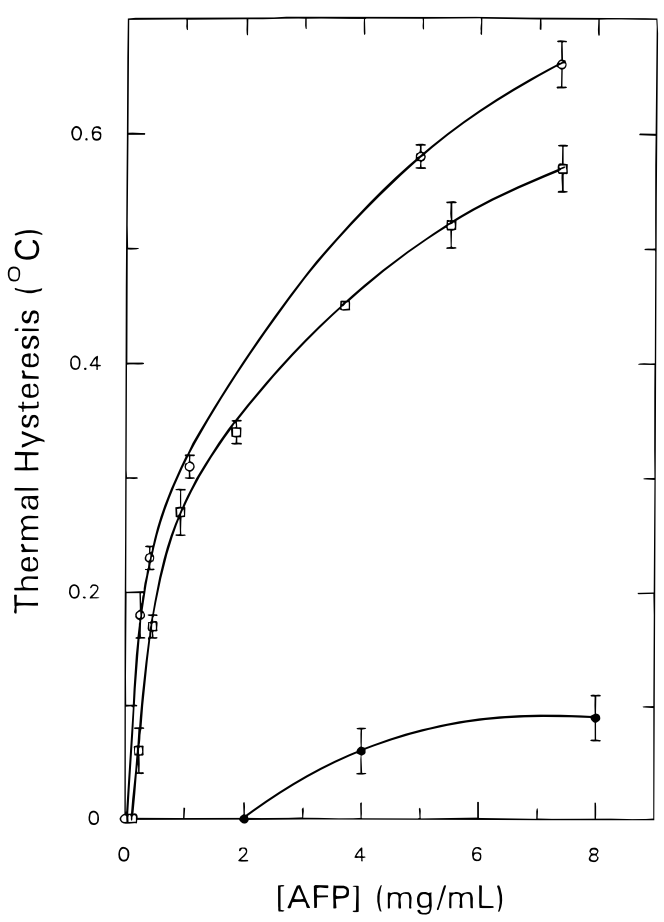

FIGURE 3: Antifreeze activity of type I AFP and its variants. Thermal hysteresis values for LTAAN (O), LVAAN ( $\square$ ), and LSAAN (-) are plotted as a function of concentration in $0.1 \mathrm{M}$ $\mathrm{NH}_{4} \mathrm{HCO}_{3}$ (pH 7.9). Each data point represents the mean of at least three determinations. Standard deviations are shown as vertical bars.

-type AFP was, as previously reported (15), indistinguishable from that of the natural protein from flounder serum (Figure 3). At a concentration of $2 \mathrm{mg} / \mathrm{mL}$ the wild-type LTAAN had a thermal hysteresis activity of $0.40{ }^{\circ} \mathrm{C}$. In marked contrast to this, LSAAN at concentrations up to $2 \mathrm{mg} / \mathrm{mL}$ was completely unable to halt the growth of a seed ice crystal. Above $2 \mathrm{mg} / \mathrm{mL}$ there was a small but measurable thermal hysteresis (up to $0.1^{\circ} \mathrm{C}$ ) within which the ice crystal was held as a hexagonal bipyramid with an average $c$ to $a$ axis ratio of 3.2 to 1 (Figure 4 ). This ice crystal was indistinguishable from that obtained with the wild-type AFP. 


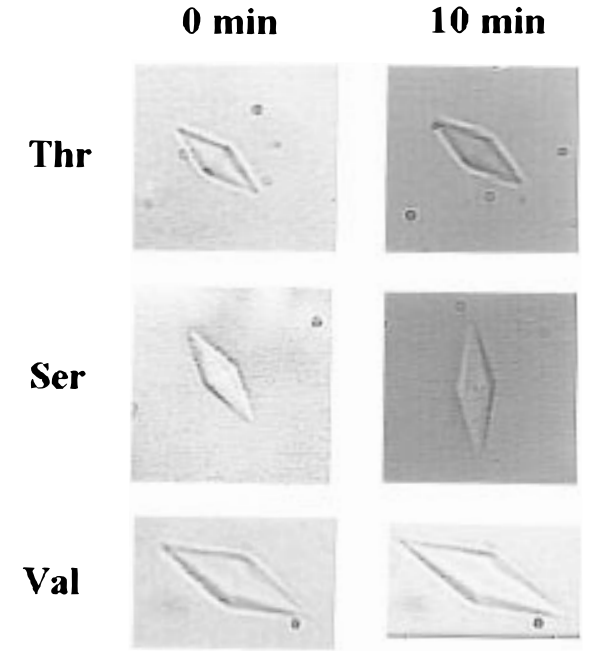

FIGURE 4: Video microscopy of ice crystals in the presence of type I AFP and variants. Ice crystals were formed in the presence of 1 $\mathrm{mg} / \mathrm{mL}$ peptide (or $16 \mathrm{mg} / \mathrm{mL}$ for LSAAN) in $0.1 \mathrm{M} \mathrm{NH}_{4} \mathrm{HCO}_{3}$ (pH 7.9) with an undercooling of $0.1{ }^{\circ} \mathrm{C}$. Still images at the start (0 $\mathrm{min})$ and the end $(10 \mathrm{~min})$ of the undercooling trial are shown for the wild-type (Thr), LSAAN (Ser), and LVAAN (Val) protein.

In contrast, the variant LVAAN was extremely active at depressing the nonequilibrium freezing point. Over the concentration range tested, it had $80-90 \%$ of the thermal hysteresis activity of the wild-type AFP. This decrease was small but statistically significant over the entire range. The ice crystal formed in the presence of $1 \mathrm{mg} / \mathrm{mL}$ LVAAN also had an average $c$ to $a$ axis ratio of 3.2 to 1 (Figure 4). This crystal grew imperceptibly both during the thermal hysteresis readings as the temperature was lowered in stages, and when held at $0.1{ }^{\circ} \mathrm{C}$ below the melting point for $10 \mathrm{~min}$. In both cases growth was only observed by time-lapse video microscopy. The crystal obtained in $1 \mathrm{mg} / \mathrm{mL}$ LTAAN did not grow under either circumstance.

Sedimentation Analysis. One trivial explanation for the dramatic loss of thermal hysteresis activity seen with LSAAN could have been self-association or aggregation of the peptide in solution. To investigate this possibility the molecular weight of LSAAN was estimated by sedimentation equilibrium analysis at $5{ }^{\circ} \mathrm{C}$ (Figure 5). The equilibrium profiles from two sedimentation runs gave an estimated molecular weight of 3292. This agrees well with the calculated monomer weight of 3215. The data are, therefore, consistent with a model that describes a monomeric species in solution at a concentration up to $6.5 \mathrm{mg} / \mathrm{mL}$ (Figure $5 \mathrm{a}$ ). LVAAN also behaved as a monomer under similar conditions (Figure $5 b)$. The estimated molecular weight of LVAAN based on the sedimentation data is 3226 , which compares favorably with the expected value of 3239. These results suggest that both LSAAN and LVAAN are monomeric in solution akin to the wild-type peptide.

NMR Analysis. The NMR spectroscopic data obtained on the variants LSAAN and LVAAN at $4{ }^{\circ} \mathrm{C}$ were of excellent quality. Within the experimental error ${ }^{1} \mathrm{H}$ chemical shifts were identical to those of the wild-type HPLC-6 peptide (15). In particular, $\mathrm{H} \alpha$ resonance shifts differed by less than 0.04 ppm between the peptides. Only small shift changes (up to $0.10 \mathrm{ppm}$ ) occurred for amide chemical shifts, primarily for residues such as L12/23 and A17/28, which are sequential or steric neighbors of the exchanged residues 13 and 24 . The peptide exhibited upfield shifted $\mathrm{C} \alpha \mathrm{H}$ resonances for all sequentially assigned residues (Table 2), as well as for all alanine residues which were (with few exceptions) not sequentially assigned due to extensive resonance overlap. Helix-typical NOE connectivities such as $d_{\alpha \mathrm{N}}(i, i+3)$ and $d_{\alpha \beta}(i, i+3)$ cross peaks were present for several residues throughout the peptides, confirming its $\alpha$-helical secondary structure (Figure 6).

The stability of these small helical peptides has been proposed to originate in the presence of a salt bridge between K18 and E22 and extensive capping structures at both termini (11). Supportive observations in the solution NMR data include $J$ coupling patterns of methylene protons indicative of extended side chains for both E22 and K18 (data not shown). While the NMR data does not provide information on the C-terminal capping due to overlap of R37 $\delta$-methylene proton resonances, $J$ coupling constants and the derived high rotamer preference of D1 (Table 2) agree with the suggestions of the crystal structure and an involvement of this residue in N-terminal capping. The hydroxyl of S4, however, does not seem to be involved in such an interaction, as its preferred $\chi 1$ rotamer of +60 precludes the formation of an intramolecular hydrogen bond with the charged terminus.

With the exception of the substituted residues 13 and 24, side-chain properties are basically identical in the LVAAN, LSAAN, and wild-type LTAAN peptides. Focusing on the putative ice-binding motif or residues, NOE cross peaks were similarly observed between Leu $\left(\delta \mathrm{CH}_{3}\right)$ and Asn $\left(\delta \mathrm{NH}_{2}\right)$ side chains one helical turn apart. This indicates the presence of some interaction between the side chains of these two residues. As previously suggested (Gronwald et al., 1996), this interaction is of a somewhat transient nature, as both residues populate all $\chi 1$ rotamer conformations albeit with a preference for the -60 rotamer (Asn approximately $60 \%$, Leu 65-70\%). Interestingly, D5 is located in an identical position regarding the ice-binding repeat as the two Asn residues but lacks the preceding Leu residue, and similarly prefers the $\chi 1-60$ rotamer with $75 \%$ occupancy.

The unchanged rotamer preferences for the Asn and Leu residues as well as the lack of NOEs between these side chains and the side chain of residues 13 and 24 in all three peptides suggests the absence of any significant steric interaction between Leu, Asn and the substituted residue (Val or Ser). The side-chain properties of the latter significantly vary between the peptides. In LSAAN only two resonance frequencies were observed for the Ser residues in correlation spectra. Nevertheless, the resonances could be unambiguously assigned using NOE correlations and their intensities. Both $\mathrm{C} \beta \mathrm{H}$ protons overlap, i.e. resonate at $3.99 \mathrm{ppm}$ (Figure 7). This overlap precludes a quantitative analysis of the sidechain conformation. However, degenerate methylene proton shifts are generally taken as being indicative of the lack of a rotamer preference, i.e. the complete rotational freedom of the respective side chain. In contrast, the valine side chain in LVAAN exhibits a strong conformer preference. Despite the partial overlap of the $\mathrm{C} \alpha \mathrm{H} / \mathrm{C} \alpha \mathrm{H}$ resonances for $\mathrm{V} 13$ and V24, both coupling constants (Table 2) could be individually determined in 1D-NMR spectra as well as by deconvolution of traces (Figure 7a) through the respective cross peaks in PE-COSY and DQF-COSY data (Figure 7). Analysis of the coupling constant of $10.2 \mathrm{~Hz}$ for both residues using a threestate jump model yields a population of $72 \%$ of the trans or $\chi 1+180$ rotamer. However, this value would also be consistent with a nonrotating side chain with a $\chi 1$ angle of 

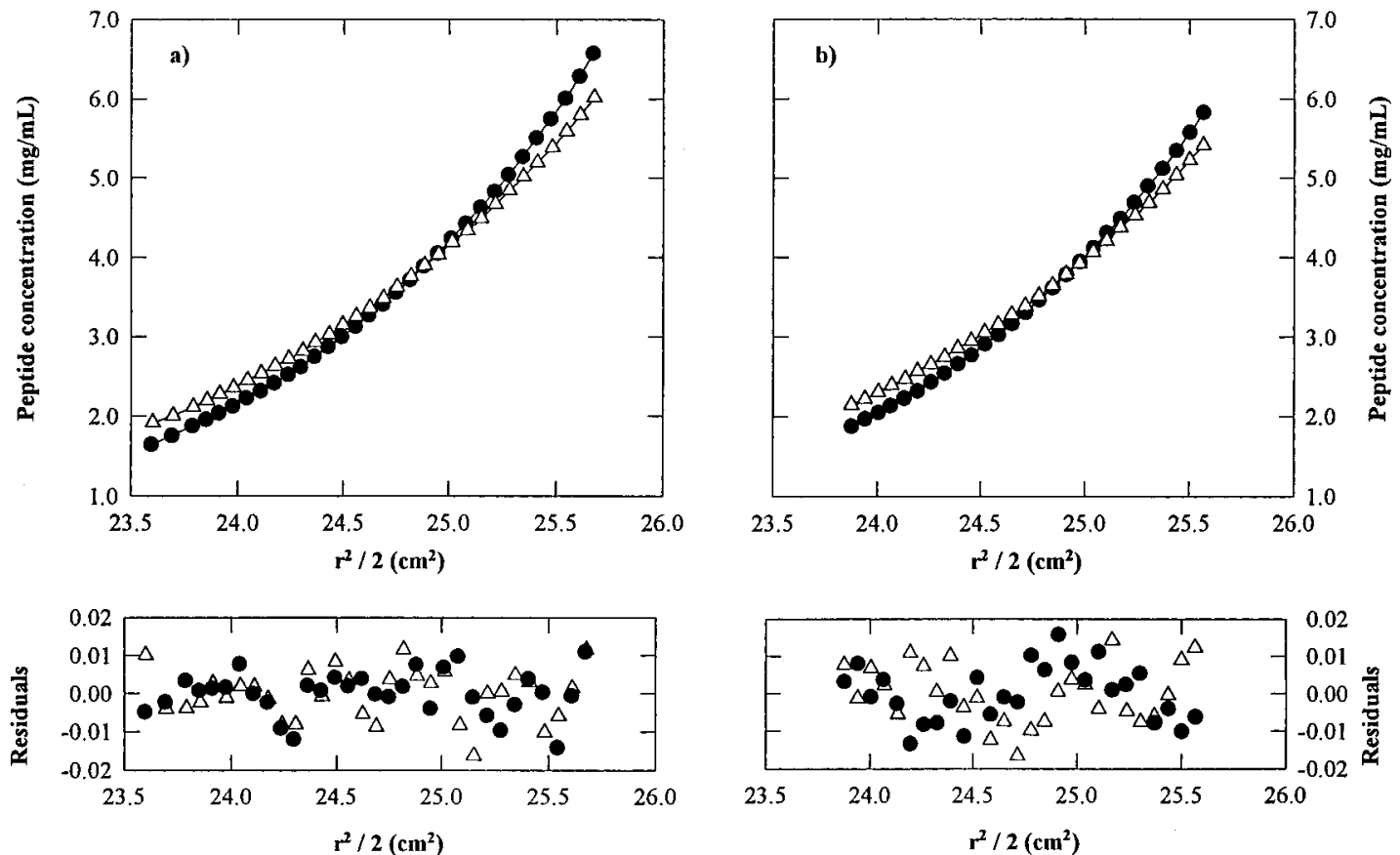

FIGURE 5: Sedimentation equilibrium studies on LSAAN. The sample was spun in $50 \mathrm{mM}$ potassium phosphate (pH 7.0 ), $50 \mathrm{mM} \mathrm{KCl}$ for $48 \mathrm{~h}$ at $5{ }^{\circ} \mathrm{C}$. The initial loading concentration was $3.52 \mathrm{mg} / \mathrm{mL}$. The run speeds were $36 \mathrm{~K}(\triangle)$ and $40 \mathrm{~K}(\bullet) \mathrm{rpm}$. The data were fitted globally by the program NONLIN. The raw data and the fitted curves for a monomer are presented in the top panel. The combined residuals of the fit are presented on the bottom panel. Symbols in the residual plot correspond to data symbols from the top panel.

\begin{tabular}{|c|c|c|c|c|c|c|c|c|c|}
\hline residue & $\delta \mathrm{NH}$ & $\delta \mathrm{HA}$ & $\delta \mathrm{HB} 2$ & $\delta \mathrm{HB} 3$ & ${ }^{3} J_{\mathrm{ab} 2}$ & ${ }^{3} J_{\mathrm{ab} 3}$ & $\%(+60)$ & $\%(-60)$ & $\%(180)$ \\
\hline \multicolumn{10}{|c|}{ Peptide LSAAN } \\
\hline $\mathrm{D} 1^{a}$ & $\mathrm{nd}^{b}$ & 4.26 & 3.16 & 2.92 & 4.30 & 5.54 & 73 & 8 & 19 \\
\hline $\mathrm{S} 4^{\mathrm{a}}$ & 8.77 & 4.31 & 4.03 & 3.92 & 4.00 & 3.90 & 89 & 8 & 19 \\
\hline D5 & 8.52 & 4.50 & 2.87 & 2.70 & 10.5 & 3.50 & 24 & 75 & 1 \\
\hline E22 & 8.35 & 4.11 & 2.07 & 2.19 & 8.20 & 5.00 & 33 & 50 & 17 \\
\hline $\mathrm{T} 2$ & nd & 4.06 & 4.15 & $-c$ & 6.60 & - & & free rotation & \\
\hline $\mathrm{L}(12 / 23)^{d}$ & $8.04 / 8.12$ & 4.31 & 1.71 & 1.86 & 10.0 & 6.0 & 3 & 70 & 27 \\
\hline $\mathrm{S}(13 / 24)$ & $8.42,8.21$ & 4.25 & 3.99 & 3.99 & nd & nd & & free rotation & \\
\hline $\mathrm{N}(16 / 27)^{d}$ & 8.73 & 4.57 & 2.97 & 2.85 & 9.00 & 4.20 & 28 & 59 & 13 \\
\hline $\mathrm{T} 35$ & 8.10 & 4.09 & 4.23 & - & 6.60 & - & & free rotation & \\
\hline \multicolumn{10}{|c|}{ Peptide LVAAN } \\
\hline $\mathrm{D} 1^{a}$ & nd & 4.29 & 3.18 & 2.94 & 4.60 & 4.00 & 83 & 13 & 4 \\
\hline $\mathrm{S} 4^{a}$ & 8.87 & 4.32 & 4.07 & 3.95 & 4.30 & 3.90 & 86 & 9 & 5 \\
\hline D5 & 8.54 & 4.52 & 2.88 & 2.72 & 9.90 & 3.90 & 27 & 68 & 5 \\
\hline E22 & 8.40 & 4.11 & 2.23 & 2.06 & nd & 4.90 & nd & & \\
\hline $\mathrm{T} 2$ & nd & 4.09 & 4.18 & - & 6.8 & - & & free rotation & \\
\hline L12 & 7.83 & 4.31 & 1.91 & 1.75 & 9.50 & 6.60 & 2 & 64 & 34 \\
\hline L23 & 7.89 & 4.33 & 1.91 & 1.75 & 9.50 & 6.60 & 2 & 64 & 34 \\
\hline V13 & 7.77 & 3.72 & 2.20 & - & 10.2 & - & \multicolumn{2}{|c|}{$28(+60$ and -60$)$} & 72 \\
\hline V24 & 7.81 & 3.73 & 2.20 & - & 10.2 & - & \multicolumn{2}{|c|}{$28(+60$ and -60$)$} & 72 \\
\hline $\mathrm{N} 16^{d}$ & 8.77 & 4.60 & 2.99 & 2.84 & 8.90 & 3.70 & 39 & 58 & 3 \\
\hline $\mathrm{N} 27^{d}$ & 8.75 & 4.58 & 2.99 & 2.84 & 8.90 & 3.70 & 39 & 58 & 3 \\
\hline T35 & 8.12 & 4.12 & 4.26 & - & 6.60 & - & \multicolumn{3}{|c|}{ free rotation } \\
\hline
\end{tabular}

${ }^{a}$ These methylene protons were not stereospecifically assigned. Thus, their chemical shift and the $-60 / 180 \chi 1$ rotamer populations are exchangeable. ${ }^{b}-$, not applicable. ${ }^{c}$ nd, not determined due to exchange, overlap or lack of intensity. ${ }^{d}$ Aliphatic resonances for residues N16/27 in LVAAN and L12/23 and N 16/27 in LSAAN completely overlap. In these cases, $J$ coupling values were obtained for the overlapped signal, assuming identical side chain conformation for these residues on the basis of the repeating sequence and complete chemical shift degeneracy.

about \pm 150 according to the appropriate Karplus angle dependence of the coupling constant (32). Thus, the valine residues in LVAAN exhibit a stronger rotamer preference than was observed for the Thr in identical positions in LTAAN, which exhibited only slight preferences of $55 \%$ for the -60 rotamer (15). It should however be noted that sterically both residue types prefer the same rotamer in these peptides, as well in helical environments in general (33). In both the $\mathrm{Thr}-60 /$ gauche $^{-}$and $\mathrm{Val}+180 /$ trans rotamer the $\mathrm{C} \beta \mathrm{H}$ proton is trans to the $\mathrm{C} \alpha \mathrm{H}$ proton, and the $\mathrm{cl}$ angle values and gauche/trans specification differ only due to the atom priority based nomenclature.

The remaining Thr side chains (2 and 35) were again flexible in the two analogs LVAAN and LSAAN. Measured coupling constants were around $6.6 \mathrm{~Hz}$ for both residues, suggesting complete rotational freedom for their side chains.

\section{DISCUSSION}

Rationale for the Design of Type I AFP Variants. Type I AFP is the least complex of the fish AFPs and the one that 


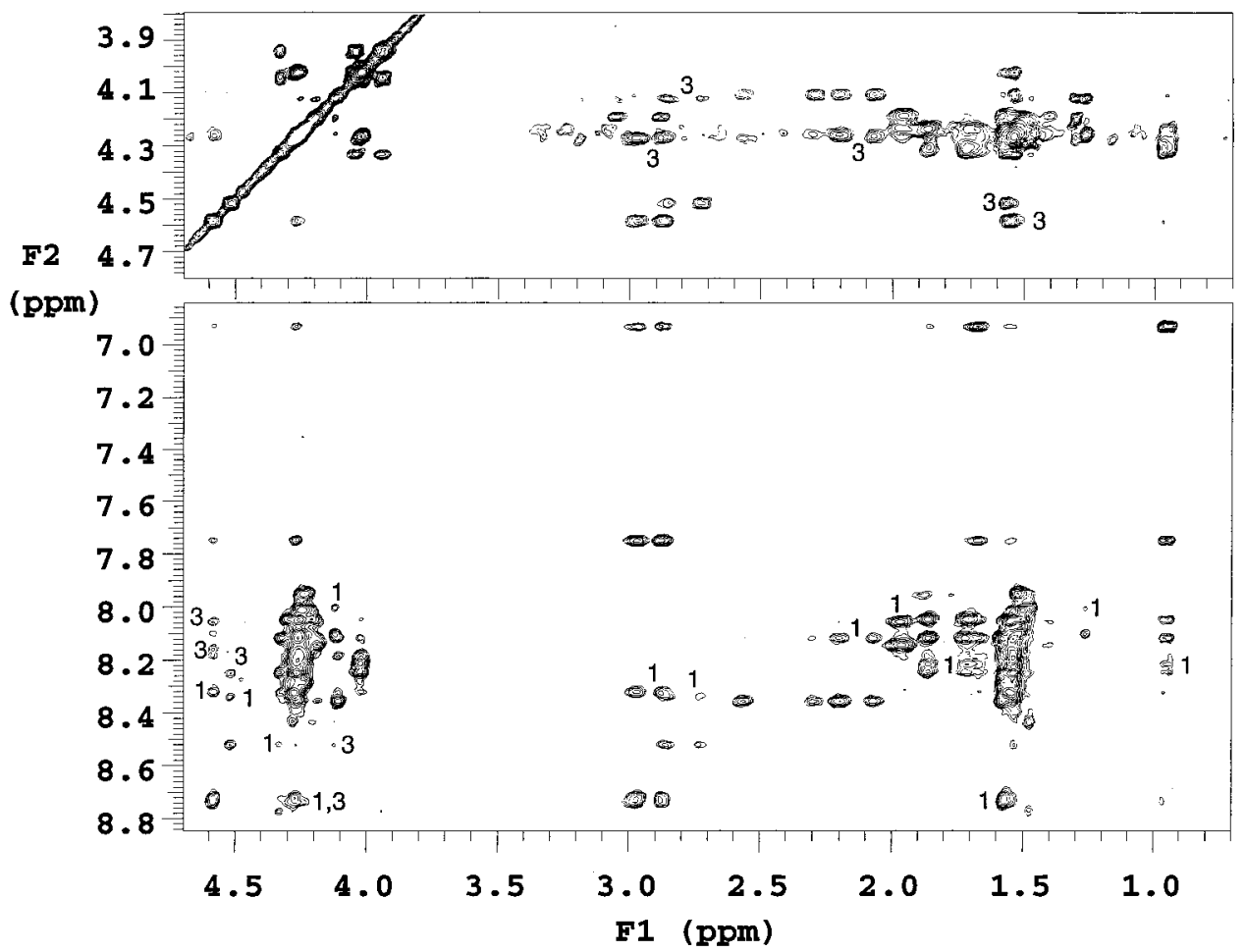

FIGURE 6: Selected regions of a ${ }^{1} \mathrm{H}-{ }^{1} \mathrm{H}-\mathrm{NOESY}$ NMR spectrum of LSAAN. The data was acquired at $4{ }^{\circ} \mathrm{C}$, pH 7.3 , using a mixing time of $100 \mathrm{~ms}$. Sequential- and helix-typical cross peaks $\left[d_{\alpha \beta}(i, i+3)\right.$ and $\left.d_{\alpha \mathrm{N}}(i, i+3)\right]$ are labeled with 1 and 3 , respectively.

is most amenable to chemical synthesis. Indeed, chemical synthesis is the only way to easily duplicate the C-terminal amidation that is an integral part of the helix C-cap. An earlier study by Wen and Laursen (34) reported on a number of synthetic variants that were designed to establish which residues were essential for binding to ice. The study targeted specific neutral and/or polar amino acids along the length of the peptide. The synthetic variants typically had lower thermal hysteresis activity than the wild-type AFP, and the authors concluded that Thr and Asn (or Asp) were critical for maximum activity. However, in most variants more that one type of amino acid replacement was made in the same molecule, and it was not possible to assess the individual impact of the different changes. We have, therefore, made a systematic replacement of Thr within the inner ice-binding motifs as designated by Sicheri and Yang (11), without changing other residues. The similarity of the two central ice-binding motifs at the start of the second and third 11amino-acid repeats allowed the same substitution to be made twice, thereby magnifying the effect of the change (Figure 1). Also, by selecting the middle two ice-binding motifs there was no chance of disrupting the internally hydrogenbonded $\mathrm{N}$ - and $\mathrm{C}$-terminal helix-cap structures.

Role of Threonine. It has always been assumed that Thr residues on winter flounder Type I AFP are largely responsible for ice binding. Their regular occupancy on one face of the helix suggests a lattice match mechanism and such a theory was advanced well before the availability of atomic resolution protein structures $(3,35)$. However, the precise form of interaction of the hydroxyl with that of the ice water layer is not defined or understood. It is assumed that the hydroxyl makes hydrogen bonds with the ice layer and provides the necessary energy of binding. From the X-ray structure, threonines in type I AFP clearly adopted a preferred $-60^{\circ}$ rotameric conformation (11). On the basis of this observation, it was suggested that Thr bind ice through a nonideal hydrogen-bonding arrangement since the hydroxyl does not protrude sufficiently from the plane of the helix. It was later confirmed by solution NMR study that Thr residues do frequently occupy the $-60^{\circ}$ state but only as one of several conformations (15). In fact, all four Thr can rotate freely and are, therefore, not conformationally restrained prior to ice binding. It is reasonable to assume that the Thr adopt a similar rotameric state once they are bound to ice, since a random distribution of all possible Thr conformations would not produce the desired lattice matching distance of $16.7 \AA$. Regardless of the rotameric state of Thr, most computational studies place these residues above the water molecules that form the ridges of the $\{20 \overline{2} 1\}$ surface (the $\{20 \overline{2} 1\}$ surface has a regularly spaced ridge and valley topology) $(8,10$, 12, 35). This seems reasonable since short $\mathrm{Thr}$ side chain is probably not able to interact directly with those water molecules that lie in the valleys. Wierzbicki et al. (36) suggested that Thr binds along the outer corner of the ridge and it is therefore partly able to continue the crystal lattice in agreement with Knight's proposal of ice incorporation of Thr. Wen and Laursen (10) proposed a similar location for Thr and suggested that Thr make at least two hydrogen bonds to two water molecules.

Relative Contributions of Thr Hydroxyl and Methyl Groups to Ice Binding. In all these studies, the contribution of Thr to ice binding is thought to be through its hydroxyl group. Yet it is interesting to observe that Ser, which should have similar hydrogen-bonding potential, is not found in the equivalent position to Thr in natural helical antifreezes (11, 17). To see if these amino acids could serve the same function, the synthetic variant was made in which two Thr were replaced by Ser. The loss of activity was catastrophic. Among the more than 20 type I analogs known, only one amino acid replacement had previously led to a complete inactivation of the protein, namely the introduction of a proline preventing helix formation (34). However, in this 
A)
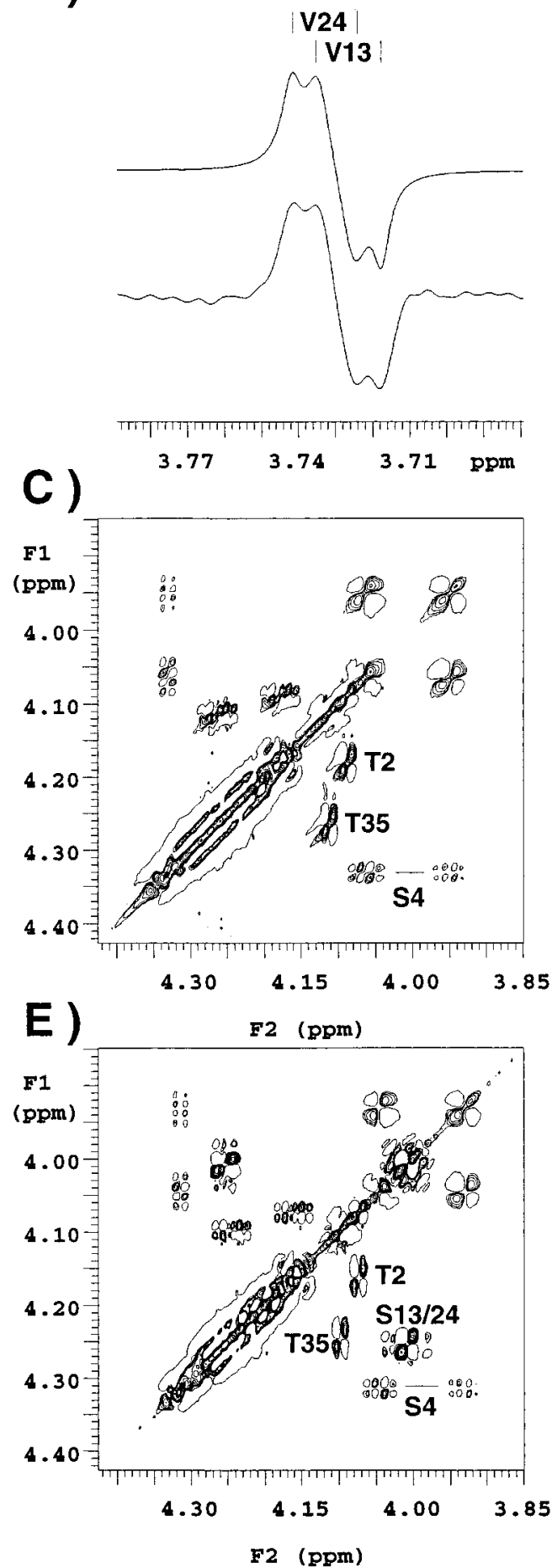

B)

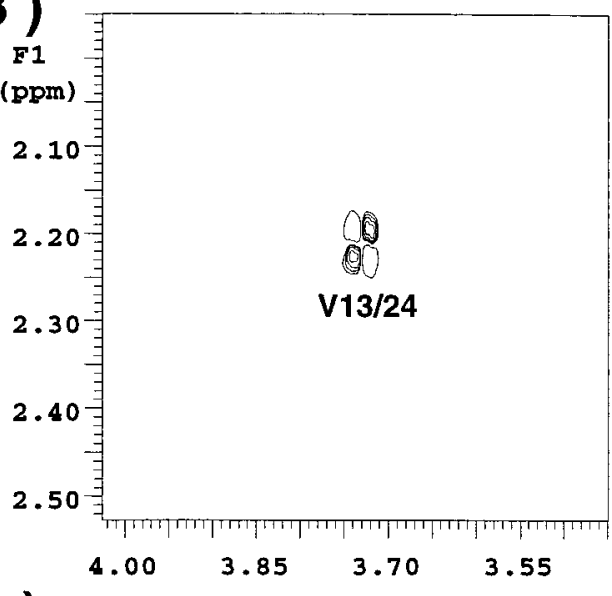

D )

F2 (ppm)

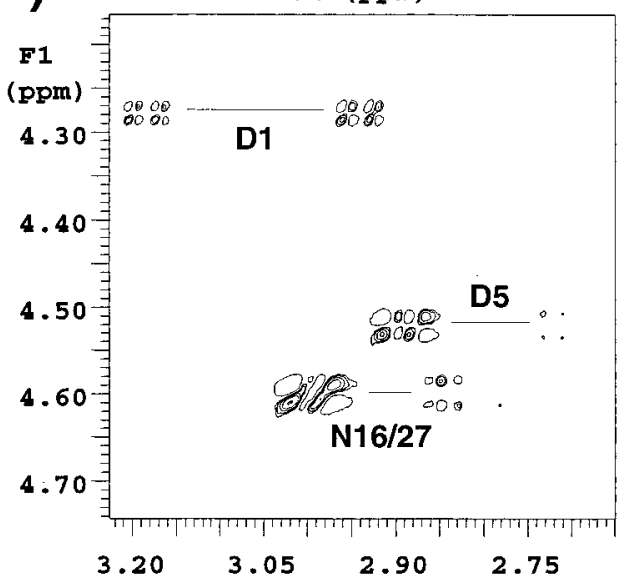

F)

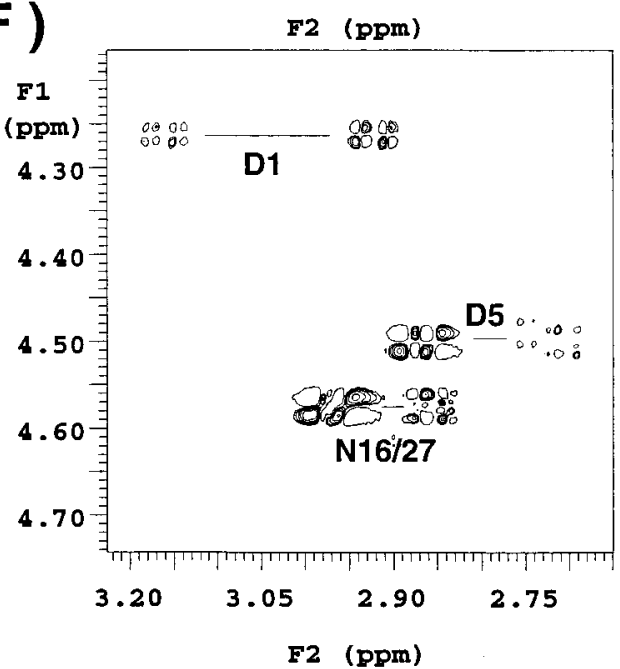

FIGURE 7: DQF-COSY NMR experiments on LSAAN and LVAAN at $4{ }^{\circ} \mathrm{C}$. Cross peaks are labeled with their single letter code and residue number. (A) Simulated and experimental traces through the $\mathrm{C} \alpha \mathrm{H} / \mathrm{C} \beta \mathrm{H}$ cross peaks of V13 and V24 in LVAAN. (B, C, and D) Regions of the aliphatic region encompassing $\mathrm{C} \alpha \mathrm{H} / \mathrm{C} \beta \mathrm{H}$ cross peaks of all non-alanine residues in LVAAN are shown. (E and F) Similar regions in the peptide LSAAN.

case trivial explanations for the loss of activity can be eliminated. The LSAAN peptide appeared to be very soluble as judged by visual inspection of the solution, a point confirmed by $\mathrm{CD}$, ultracentrifugation, and NMR. CD analysis showed that the peptide was helical, and sedimentation analysis indicated that it was not aggregated. Furthermore, during the course of this work we learned that the LSAAN peptide had also been synthesized by Haymet, Knight, and co-workers who observed a similar activity loss (A. D. J. Haymet, personal communication).
At high peptide concentrations LSAAN bound to ice and produced an ice crystal that matched the morphology of that obtained with wild-type AFP, indicating that the antifreeze was binding to the same pyramidal plane and, by inference, the same direction. Thus, the activity loss could be considered as a decrease in affinity for the ice site because it was compensated for by an increase in AFP concentration. The basis for this loss of affinity appears to be the lack of the $\gamma$-methyl group. Prior to the NMR study of Thr rotamers in type I AFP (15), one could have speculated that the basis 
for the loss of activity was that the hydroxyls were not locked into a uniform configuration that would allow simultaneous interaction (11) or zippering (8) to ice. However, this hypothesis does not seem probable based on a more indepth analysis.

It was observed that the Thr side chain has a $55 \%$ preference for the $-60^{\circ}$ rotamer (15). In comparison, the Ser side chain of the LSAAN variant showed no preference for any of the three possible rotameric configurations $\left(-60^{\circ}\right.$, $180^{\circ}$, and $60^{\circ}$ ). If the protein adsorbed to the ice exhibited only one rotamer for this side chain, an assumption based on the ice-lattice-match hydrogen-bonding hypothesis, then different contributions of conformational entropy loss would be expected for these two side chains upon adsorption. However, given the mentioned population differences, the conformational entropy loss of the Thr side chain would be only $10 \%$ smaller than that of the Ser side chain. Using data from Gomez and Freire, (37), the $\Delta \Delta S$ can be estimated to be $<0.2 \mathrm{kcal} / \mathrm{mol}$. In addition, the conformational energy differences between the Thr rotamers are neglible; a preference of $55 \%$ indicates that the $-60^{\circ}$ rotamer would favor the ice adsorption by $<0.5 \mathrm{kcal} / \mathrm{mol}$ (only if the bound conformation was also $-60^{\circ}$ ). Thus, while the differences in rotamer populations between the Ser and Thr variants will have small energetic consequences, their magnitude $(<1 \mathrm{kcal} /$ mol per Thr substitution) is insufficient to explain the observed difference in antifreeze activity, in particular as the binding of the wild-type/Thr analog is generally perceived to be very high affinity or irreversible. Furthermore, from a kinetic viewpoint, it is unlikely that the rotamer population differences could affect the protein's activity. The NMR results indicate that rotational barriers for side chain rotation are low for both side chains, and the conformers interconvert rapidly especially when this is compared to the limiting diffusion rate of the protein in solution during the adsorption event.

The importance of the $\gamma$-methyl group on T13 and T24 was confirmed by the restoration of $80-90 \%$ activity in LVAAN. What was equally surprising about this variant was that the replacement of the hydroxyl group by a methyl group did not cause much loss in activity in a molecule that traditionally has been thought to have too few hydrogen bonds to bind tightly to ice. This result provides the first plausible evidence for an AFP ice-binding hypothesis that places an increased emphasis on the importance of van der Waals interactions between AFP and ice (38). Val is a very good space-filling match for Thr (Figure 1), and both residues exhibit sterically similar side chain rotamer preferences in these peptides and in general. Thus, the steric complementarity of the protein and ice surface are not compromised, leading to comparable van der Waals interactions of wildtype and LVAAN variant with the ice surface. Van der Waals interactions have been previously used to explain a $33 \%$ loss of antifreeze activity when L12 and L23 were replaced by alanine (34).

In addition, significant favorable entropic contributions are likely to arise from the desolvation of protein and ice surfaces upon the protein binding to ice. The Thr-Val substitution will to some extent change the polar/nonpolar ratio of the protein surface that becomes buried upon protein-ice binding and thus affect this contribution. However, the L12A + L23A replacements (34) and the T13S + T24S mutations reported here will more significantly affect these entropic contributions. Not only will the protein's buried nonpolar and total accessible surface area be reduced upon binding, but the loss of surface complementarity could lead to less efficient solvent shielding of the interface (or protein-ice hydrogen bonds therein) and consequentially to a reduction of the total buried surface area and solvent related entropy gain.

Although it seems likely that hydrogen bonds contribute to the binding of AFP, if only due to the observed residual ice crystal growth with LVAAN and the slightly higher activity of LTAAN, in a thermodynamic analysis other differences such as the changed non-polar and polar surface areas would have to be considered for their enthalpic and entropic consequences. In conclusion, the described results certainly call into question the dominance of hydrogen-bond interactions in the protein-ice adsorption, but their importance, at least in absolute energetic terms, is not necessarily changed. The energetic contributions of hydrogen bonds can be as significant (or small) as previously proposed. It is however clear that other energetic contributions of at least similar magnitude exist and originate in nonpolar interactions in the protein-ice-water system.

\section{ACKNOWLEDGMENT}

We thank Paul Semchuk, Iain Wilson, and Leonard Daniels for peptide synthesis and mass spectrometry, the Alberta Peptide Institute for amino acid analyses, Les Hicks for ultracentrifugation analysis, and D. Venkat Reddy and Andrea Cserey for their contributions to the NMR study. We are grateful to Tony Haymet and Charles Knight for making available to us a preprint of their paper (J. Am. Chem. Soc. JA951427M-136) describing serine-substituted type I AFP and to Pierre Lavigne for helpful discussions.

\section{REFERENCES}

1. Davies, P. L., and Hew, C. L. (1990) FASEB J. 4, 24602468.

2. Yang, D. S. C., Sax, M., Chakrabartty, A., and Hew, C. L. (1988) Nature 333, 232-237.

3. DeVries, A. L., and Lin, Y. (1977) Biochim. Biophys. Acta 495, 388-392.

4. Raymond, J. A., and DeVries, A. L. (1977) Proc. Natl. Acad. Sci. U.S.A. 74, 2589-2593.

5. Knight, C. A., Cheng, C. C., and DeVries, A. L. (1991) Biophys. J. 59, 409-418.

6. Wilson, P. W. (1993) Cryo-Lett. 14, 31-36.

7. Laursen, R. A., Wen, D., and Knight, C. A. (1994) J. Am. Chem. Soc. 116, 12057-12058.

8. Chou, K.-C. (1992). J. Mol. Biol. 223, 509-517.

9. Jorgensen, H., Mori, M., Matsui, H., Kanaoka, M., Yanagi, H., Yabusaki, Y., and Kikuzono, Y. (1993) Protein Eng. 6, 19-27.

10. Wen, D., and Laursen, R. A. (1992) Biophys. J. 63, 16591662.

11. Sicheri, F., and Yang, D. S. C. (1995) Nature 375, 427-431.

12. Madura, J. D., Wierzbicki, A., Harrington, J. P., Maughon, R. H., Raymon, J. A., and Sikes, C. S. (1994) J. Am. Chem. Soc. 116, 417-418.

13. Knight, C. A., Driggers, E., and DeVries, A. L. (1993) Biophys. J. 64, 252-259.

14. Chao, H., DeLuca, C. I., and Davies, P. L. (1995) FEBS Lett 357, 183-186.

15. Gronwald, W., Chao, H., Reddy, D. V., Davies, P. L., Sykes, B. D., and Sönnichsen, F. D. (1996) Biochemistry 35, 1669816704.

16. Hodges, R. S., Semchuk, P. D., Taneja, A. K., Kay, C. M., Parker, J. M. R., and Mant C. T. (1988) Pept. Res. 1, 19-30. 
17. Chao, H., Hodges, R. S., Kay, C. M., Gauthier, S. Y., and Davies, P. L. (1996) Protein Sci. 5, 1150-1156.

18. Johnson, M. L., Correia, J. J., Yphantis, D. A., and Halvorson, H. R. (1981) Biophys. J. 36, 575-588.

19. Wishart, D. S., Boyko, R. F., Willard, L., Richards, F. M., and Sykes, B. D. (1994) Comput. Appl. Biosci. 10, 121-132.

20. Cohn, E. J., and Edsall, J. T. (1943) Proteins, Amino Acids and Peptides as Ions and Dipolar Ions Reinhold, New York.

21. Chakrabartty, A., and Hew, C. L. (1991) Eur. J. Biochem. 202, 1057-1063.

22. DeLuca, C. I., Chao, H., Sönnichsen, F. D., Sykes, B. D., and Davies, P. L. (1996) Biophys. J. 71, 2345-2355.

23. Piantini, U., Sorensen, O. W., and Ernst, R. R. (1982) J. Am. Chem. Soc. 104, 6800-6801.

24. Rance, M., Sorensen, O. W., Bodenhausen, G., Wagner, G., Ernst, R. R., and Wüthrich, K. (1983) Biochem. Biophys. Res. Commun. 117, 479-485.

25. Jeener, J., Meier, B. H., Bachmann, P., and Ernst, R. R. (1979) J. Chem. Phys. 71, 4546-4553.

26. Macura, S., and Ernst, R. R. (1980) Mol. Phys. 41,95-117.

27. Davis, D. G., and Bax, A. (1985) J. Am. Chem. Soc. 107, 2820-2820.

28. Piotto, M., Saudek, V., and Sklenar, V. (1992) J. Biomol. NMR $2,661-665$.
29. Mueller, L. (1987) J. Magn. Reson. 72, 191-196.

30. Chakrabartty, A., Kortemme, T., and Baldwin, R. L. (1994) Protein Sci. 3, 843-852.

31. Zhou, N. E., Monera, O. D., Kay, C. M., and Hodges, R. S. (1994). Protein Pept. Lett. 1, 114-119.

32. DeMarco, A., Llinas, M., and Wüthrich, K. (1978) Biopolymers 17, 617-636.

33. Dunbrack, R. L, Jr., and Karplus, M. (1993) J. Mol. Biol. 230, 543-574.

34. Wen, D., and Laursen, R. A. (1992) J. Biol. Chem. 267, 14102-14108.

35. DeVries, A. L. (1984) Philos. Trans. R. Soc. London, Ser. B B304, 575-588.

36. Wierzbicki, A., Taylor, M. S., Knight, C. A., Madura, J. D., Harrington, J. P., and Sikes, C. S. (1996) Biophys. J. 71, 8-18.

37. Gomez, J., and Freire, E. (1995) J. Mol. Biol. 252, 337-350.

38. Sönnichsen, F. D., DeLuca, C. I., Davies, P. L., and Sykes, B. D. (1996) Structure 4, 1325-1337.

39. Scholtz, J. M., Qian, H., York, E. J., Stewart, J. M., and Baldwin, R. L. (1991) Biopolymers 31, 1463-1470.

BI970817D 